\title{
STM/STS Studies of Self-Organized Growth of Iron Silicide Nanocrystals on Vicinal Si(111) Surface
}

\author{
A. WhWro ${ }^{a, b}, \mathrm{~S} \cdot \mathrm{Suto}^{c}, \mathrm{R} \cdot \mathrm{CzaJKA}^{a, d}$ AND A. KasuYA ${ }^{a}$ \\ ${ }^{a}$ Center for Interdisciplinary Research, Tohoku University \\ Sendai 980-8578, Japan \\ ${ }^{b}$ Institute of Physics, Polish Academy of Sciences \\ al. Lotników 32/46, 02-668 Warszawa, Poland \\ ${ }^{c}$ Department of Physics, Graduate School of Science \\ Tohoku University, Sendai 980-8578, Japan \\ ${ }^{d}$ Faculty of Technical Physics, Poznań University of Technology \\ Nieszawska 13A, 60-965 Poznań, Poland
}

\begin{abstract}
The evolution of iron silicide structure grown by solid state epitaxy on $\mathrm{Si}(111)$ vicinal surface was investigated by scanning tunnelling microscopy. The reactions, which occur on the surface, are compared for two various $\mathrm{Fe}$ coverages: 0.33 and 2 monolayers. The annealing at $250^{\circ} \mathrm{C}$ does not enable substantial recovery of the surface ordering, deteriorated by Fe deposition at room temperature. The onset of $2 \times 2$ surface reconstruction is observed upon annealing at $400^{\circ} \mathrm{C}$. A three-dimensional growth tendency of iron silicide crystallites on a bare $\mathrm{Si}(111) 7 \times 7$ surface was found at $700^{\circ} \mathrm{C}$. In the case of 2 monolayer coverage crystallites nucleate along the edges of substrate terraces forming a regular array of nanometer size dots. Basing on atomically resolved spectroscopic effects and statistical considerations, structure of iron silicide nanocrystallites as well as Schottky-like character of the barrier at the interface between metallic crystallite and semiconducting substrate is deduced.
\end{abstract}

PACS numbers: $61.46 .+\mathrm{w}, 68.35 .-\mathrm{p}, 68.37 . \mathrm{Ef}, 81.10 . \mathrm{Jt}$

\section{Introduction}

Iron silicide has attracted much attention because of the complexity of numerous compounds and serious expectations for practical applications [1]. De- 
pending on the crystalline structure it reveals various properties: semiconducting $\left(\beta-\mathrm{FeSi}_{2}\right)$, metallic $\left(\alpha-\mathrm{FeSi}_{2}, \varepsilon-\mathrm{FeSi}\right)$ or ferromagnetic $\left(\mathrm{Fe}_{3} \mathrm{Si}\right)$. Particularly the semiconducting phase is a very promising material for Si-based optoelectronic devices, because it has a narrow energy gap of $0.87 \mathrm{eV}$, which corresponds to the maximum transmission of the optical glass fibers. Moreover, compounds grown in the form of thin films reveal even wider group of structures, some of which have no counterpart in the bulk. The phases, which do not appear in a bulk material, are stabilized by the strains, generated by the lattice mismatch at the interface with the $\mathrm{Si}$ substrate. The grown structures are very sensitive to the various deposition methods: co-deposition by molecular beam epitaxy [2-7], reactive deposition epitaxy [8-10], solid state epitaxy [11-16] as well as to parameters which affect kinetic factors. Therefore, apparently the same growth conditions may result in substantially different phases displaying undesirable properties.

Iron silicide phase transition upon annealing is another contradictory issue. The phase transition temperature is affected by the film thickness, because the factors, which stabilize epitaxial phases, significantly depend on the interface structure $[2,3,7,9,13,15,17-19]$.

The composition of the topmost atomic layer is also intensively discussed matter. It is generally accepted that the termination layer consists of $\mathrm{Si}$ atoms $[11,14,20,21]$, because they lower the surface energy of iron silicide. However, sparse experimental works and theoretical considerations reveal the presence of $\mathrm{Fe}$ atoms on the surface $[22,23]$.

The early stage formation of iron silicide was the aim of our investigations. The reactions, which occur upon low coverage $\mathrm{Fe}$ deposition onto Si(111) vicinal surface, activated by subsequent successive annealing, were studied with atomic resolution by means of scanning tunnelling microscopy (STM), supported by spectroscopic measurements. Our attention was also focused on the influence of vicinal structure of the substrate on surface diffusion, which affected the growth mode of iron silicide.

\section{Experimental}

Iron was deposited on silicon at room temperature in a UHV system at the base pressure in the low range of $10^{-10}$ Torr. The sample substrates were cut from commercial $n$-type $\mathrm{Si}(111)$ wafers (Sb doped, $\approx 1 \Omega \mathrm{cm}$ ), misoriented by $1.2^{\circ}$ in $\left[\begin{array}{lll}-1 & -1 & 2\end{array}\right]$ direction. Clean surface was achieved through heating at $700^{\circ} \mathrm{C}$ for several hours and flashing at $1250^{\circ} \mathrm{C}$ up to 5 times, followed by cooling down at the rate lower than $1^{\circ} \mathrm{C} / \mathrm{s}$. Such thermal treatment resulted in the high quality surface showing $7 \times 7$ reconstruction with a negligible amount of defects and contamination. As a consequence of cutting misorientation the substrate surface had a terrace-like structure $800-1000 \AA$ in width, separated by edge steps about $20 \AA$ in average height. Iron was sublimated onto the substrate from a tungsten 
basket typically at a rate of $5 \AA / \mathrm{min}$. The deposition rates and the thickness were monitored by a quartz crystal microbalance. It was assumed that 1 monolayer (ML) corresponded to $1.2 \times 10^{15}$ atoms $/ \mathrm{cm}^{2}$, i.e. average value of the low index surfaces of bcc Fe. The scanning tunnelling microscopy measurements were performed in the same vacuum system at room temperature directly after iron deposition and subsequent annealing for $10 \mathrm{~min}$ at: $250^{\circ} \mathrm{C}, 400^{\circ} \mathrm{C}$, and $700^{\circ} \mathrm{C}$. The STM tips were electrochemically etched from a tungsten wire $(0.2 \mathrm{~mm}$ in diameter $)$ and cleaned by annealing in the vacuum system. The sample was biased relatively to the tip in our setup. This means that the empty states were imaged for positive voltage, whereas the occupied ones were probed for negative bias. All STM topographic images were recorded in the constant current mode.

\section{Results and discussion}

The deposition of Fe on Si substrate deteriorates, in general, the reconstruction observed for clean surface. In the case of $0.33 \mathrm{ML}$ Fe deposition, clusters of about one atomic monolayer in height and $1 \mathrm{~nm}$ in diameter randomly appear on the surface. Single atoms, irregularly aligned between them, are visible, however their unambiguous identification on the basis of the topographic image is questionable. Occasionally, small fragments of non-destroyed Si substrate with remnant $7 \times 7$ reconstruction are found. Upon deposition of $2 \mathrm{ML}$ Fe larger agglomerates, of non-regular shape and few nanometers in diameter, are formed. The surface between them does not reveal the ordering typical of clean Si surface anymore. Results achieved for Fe are in contrast with an experiment performed in the same conditions for Au deposits. After deposition of Au submonolayer on Si(111) surface, small clusters nucleate mainly in the centres of both halves of the unit meshes [24]. A clear $7 \times 7$ substrate reconstruction visible between clusters suggests that the deposited Au does not react with Si. Therefore our findings allow an inference that the solid state reaction between the deposited iron and the silicon substrate proceeds intensively already at room temperature.

A short-range ordering of surface atoms becomes visible after annealing at $250^{\circ} \mathrm{C}$. Three atoms' rings randomly distributed are the only regular objects encountered on the surface. However, their azimuthal planar orientation is identical, suggesting the influence of the Si substrate symmetry on their formation process. The distance between atoms, being members of a ring, equals to $6.6 \AA$, which is much more than the bonding length in the bulk Si $(3.86 \AA)$. Therefore the observed ordering cannot be considered as $1 \times 1$ reconstruction $[4,14,25]$. It rather correlates well with $2 \times 2$ reconstruction of $\varepsilon$ phase [26], however this phase is not found upon annealing at higher temperatures. Thus the formation of the rings might be associated with the initial stage of ( $\mathrm{CsCl}$ )FeSi phase growth, unambiguously identified after annealing at higher temperature.

Upon annealing at $400^{\circ} \mathrm{C}$ the long-range ordering of the surface is activated. The proceeding processes drastically depend on the amount of deposited material. 
The reconstruction of the surface is not completed for the coverage of $0.33 \mathrm{ML}$ Fe. Well-ordered islands are separated by areas, where the atoms display merely short-range ordering similar to that observed after annealing at $250^{\circ} \mathrm{C}$ (Fig. 1). All characteristic features i.e. three atoms rings with the distance between member atoms equal to $6.6 \AA$, random positions of rings on the surface and their identical azimuthal orientation are found after annealing at $400^{\circ} \mathrm{C}$. The amount of three atoms rings is substantially higher in comparison with treatment at lower temperature. The surface of ordered islands, several nanometers in size, is terminated with identically imaged by STM atoms aligned in hexagonal, closely packed structure. The nearest neighbour distance, equal to $7.6 \AA$, corresponds to $2 \times 2$ reconstruction of $\mathrm{CsCl}$ - or $\mathrm{CaF}_{2}$-type phase of iron silicide. The islands, which usually have non-regular shape, are located randomly on the substrate surface. The same azimuthal orientation of all islands proves their epitaxial growth on $\mathrm{Si}$ surface.

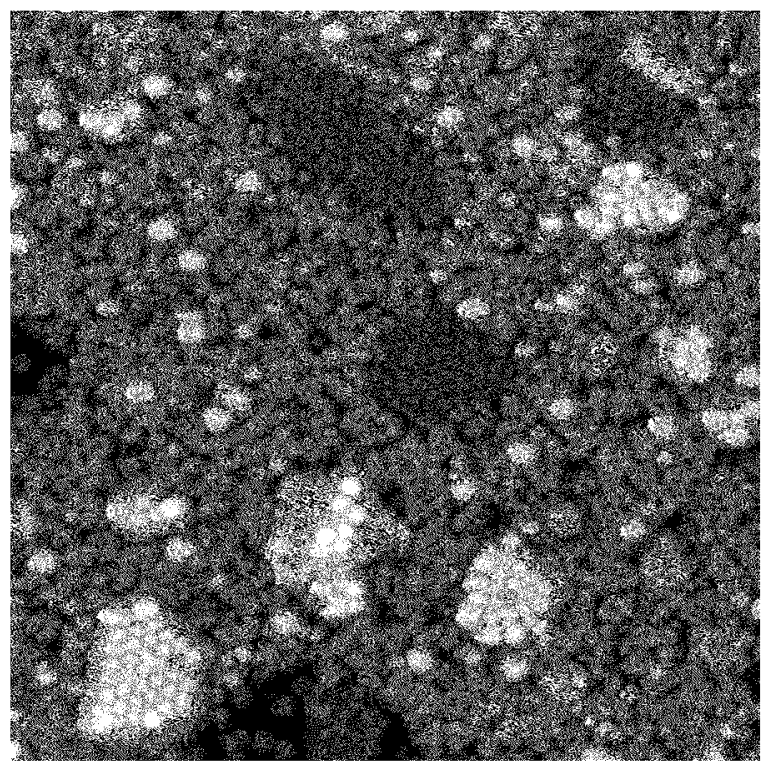

Fig. 1. Partial $2 \times 2$ reconstruction upon deposition of $0.33 \mathrm{ML}$ Fe and annealing at $400^{\circ} \mathrm{C}\left(37.5 \times 37.5 \mathrm{~nm}^{2}\right)$.

Deposition of $2 \mathrm{ML}$ of $\mathrm{Fe}$ annealed subsequently at $400^{\circ} \mathrm{C}$, on the contrary to the discussed above case, results in complete surface ordering. Large scan STM image (Fig. 2) shows three entirely reconstructed terraces of Si vicinal substrate. The roughness of each terrace is very low - regular in shapes, atomically flat areas are confined by borders oriented every $60^{\circ}$, determined by the rows of atoms. The fluctuation of the surface profile within the same terrace does not exceed one monolayer. Figure 3a shows a high resolution STM topography image of a single 


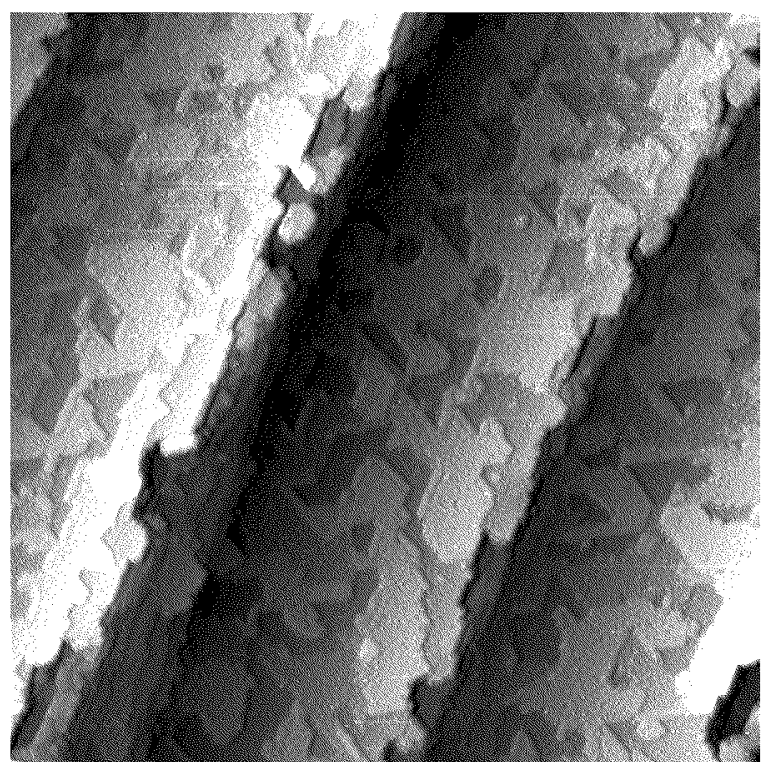

Fig. 2. The topography image with derivative component $\left(200 \times 200 \mathrm{~nm}^{2}\right)$ of entirely reconstructed vicinal surface covered with $2 \mathrm{ML} \mathrm{Fe}$ and annealed at $400^{\circ} \mathrm{C}$.

terrace. The image was recorded at negative bias, for which the occupied states were probed. Three terminating atomic planes are clearly distinguished. The STM image of surface atoms differs depending on the plane. In the lowest layer every atom seems to be identical. In the middle plane some atoms are seen as the "lower" ones (for further discussion they are assumed to be of A-type, whereas the others - as B-type). As discussed in the next part of the paper, variation in apparent height of surface atoms imaged by STM is associated merely with spectroscopic effects, which suggest that their electronic structures differ. Atoms, imaged as "lower", are in minority and their population is estimated on the level of $12 \%$. Their distribution in plane is random, without any tendency to agglomerate in clusters. In the highest plane the contribution of A-type and B-type atoms is comparable, therefore it frequently happens that two or more atoms of the same type neighbour each other. The surface profile (Fig. 3b) enables unambiguous differentiation of two types of atoms. The height of terrace steps, equal to $1.5 \AA$, favours CsCl-type structure of iron silicide over $\mathrm{CaF}_{2}$-type, for which the terrace step height is expected to be equal to $3.1 \AA$ [2]. On this basis one may infer that all atoms in the lowest plane are of B-type, which type appears also in majority in the middle terrace and finally in comparable amount to A-type atoms in the highest plane. The distribution trend of both types of atoms on the surface the higher terrace the higher concentration of A-type atoms - is very clear. For negative bias A-type atoms are imaged as "lower", as mentioned previously, by $0.6 \AA$. 

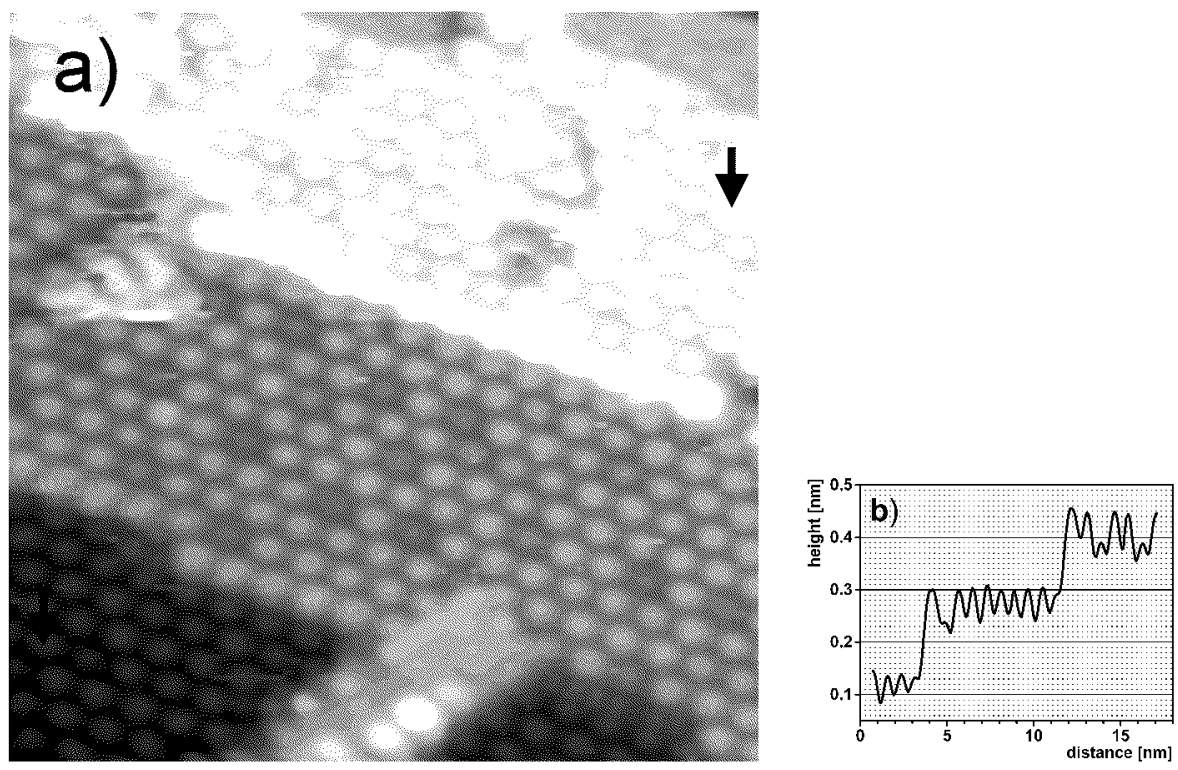

Fig. 3. (a) Recorded at $-1.93 \mathrm{~V}$ STM image $\left(15 \times 15 \mathrm{~nm}^{2}\right)$ of a single vicinal terrace after deposition of $2 \mathrm{ML} \mathrm{Fe}$ and subsequent annealing at $400^{\circ} \mathrm{C}$. The content of atoms imaged as "lower" (A-type) depends on the terminating atomic plane with a tendency: higher plane-higher contribution, (b) the surface profile measured between arrows.

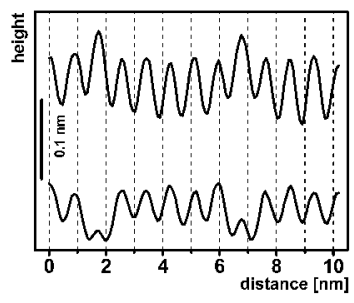

Fig. 4. Corrugation profile of the same row of atoms taken at $+1.9 \mathrm{~V}$ (upper) and $-1.9 \mathrm{~V}$ (bottom).

Typical variation of the atom imaging by STM is quantitatively depicted in Fig. 4, which shows two corrugation profiles along the row of atoms, containing both $\mathrm{A}$ and $\mathrm{B}$ types, measured at various biases. In the upper profile, where the empty states are probed, the apparent height of $A$-type atoms is larger by $0.2 \AA$ relatively to B-type ones. The opposite bias inverts the STM image - the A-type atoms are probed as "lower", approximately by $0.4 \AA$ in comparison to B-type ones. The neighbourhood of different in type atoms does not seem to affect the way of imaging. As seen in the profile, B-type atoms adjacent to A-type have the same apparent height as these in the uniform row of B-type atoms. The above lack 
of neighbourhood influence may be a consequence of weak interaction in plane resulting from the large separation distance and/or a strong localization of electronic states. Also the absence of lateral shift position of atoms imaged at opposite biases suggests the lack of spatial separation of empty and occupied states.

The annealing at higher temperature activates entirely different growth mode of iron silicide. The growth of iron silicide at $700^{\circ} \mathrm{C}$ is no longer two-dimensional, as at lower temperatures. Instead, three-dimensional nanometer size crystallites are formed on the bare Si $7 \times 7$ reconstructed surface. Their size, shape, and location on the surface substantially depend on the amount of the deposited material.

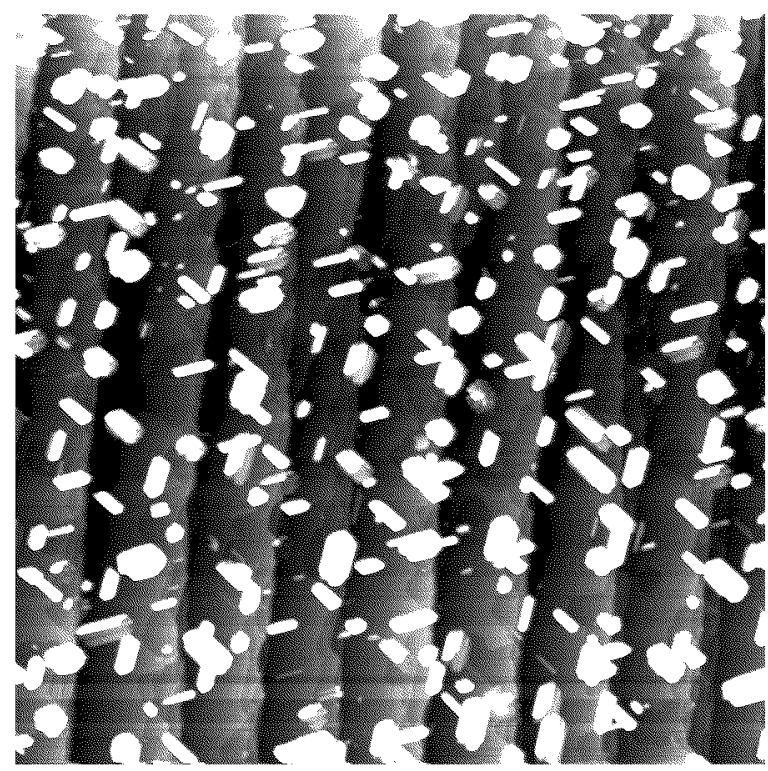

Fig. 5. Three-dimensional iron silicide crystallites grown upon deposition of 0.33 ML Fe and annealing at $700^{\circ} \mathrm{C}\left(\operatorname{scan}\right.$ size: $\left.1 \times 1 \mu \mathrm{m}^{2}\right)$.

Crystallites formed upon $0.33 \mathrm{ML}$ coverage are homogeneously distributed - their position on the surface is not affected by the edges of vicinal structure (Fig. 5). They nucleate both in the vicinity of the edges as well as in the centre of terraces. Prevailing number of crystallites have an elongated shape. The multitude of such forms may suggest that they are the initial stage of three-dimensional iron silicide crystallites at low Fe coverage regime. However, a certain amount of non-regular crystallites is also encountered. The long axes of crystallites clearly determine three directions, oriented every $60^{\circ}$, reflecting the symmetry of $\operatorname{Si}(111)$ $7 \times 7$ surface.

Figure 6 shows a high resolution STM image of a typical crystallite taken at a bias of $-2.27 \mathrm{~V}$. The edges of the base are parallel to [-110] directions of the unit mesh of the well reconstructed Si substrate. The length of the crystallite 


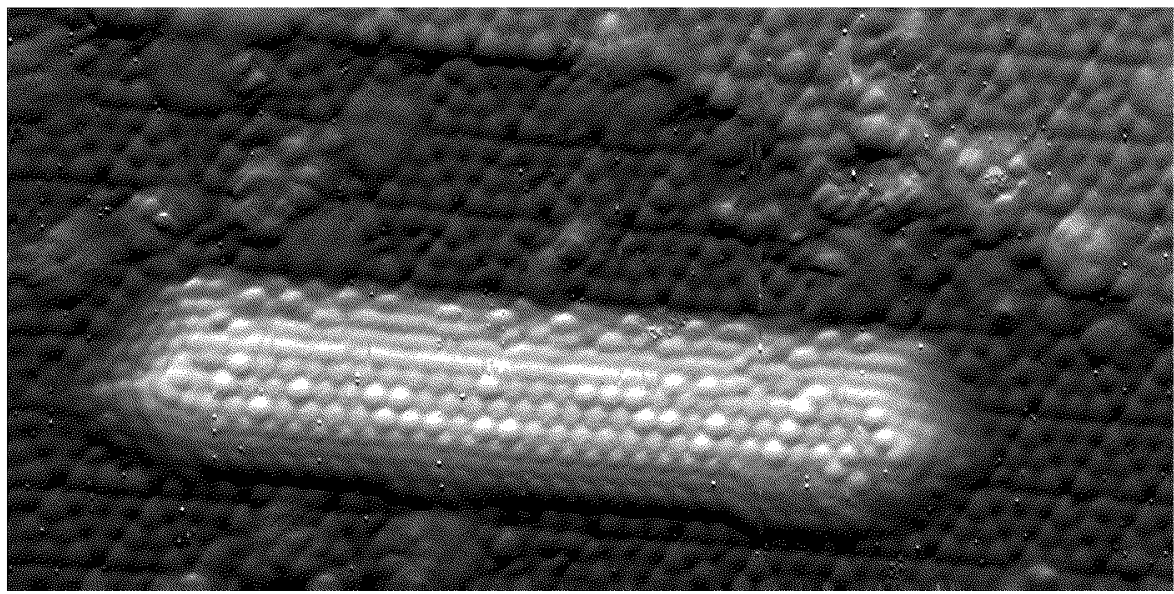

Fig. 6. Taken at $-2.27 \mathrm{~V}$, high resolution $\left(20 \times 40 \mathrm{~nm}^{2}\right)$ derivative image of a single iron silicide crystallite nucleated after deposition of $0.33 \mathrm{ML}$ Fe and subsequent annealing at $700^{\circ} \mathrm{C}$.

is about $250 \AA$. The topmost layer, three atomic rows in width, shows a $(2 \times 2)$ reconstruction with the distance between nearest neighbour atoms equal to $7.6 \AA$, which is expected both for $\mathrm{CsCl}$ - and $\mathrm{CaF}_{2}$-type structure of iron silicide $[2,11,27]$. However, the excellent quality of the image allows finding that the crystallite consists of five monolayers, which in combination with its height equal to $7.9 \AA$ reveals that it has a structure of CsCl-type. Various imaging of atoms in the topmost layer and on the facets, similar to that observed upon annealing at lower temperature (see Fig. 3a), as well as different apparent height of the crystallite measured at different biases, have a spectroscopic nature and will be discussed in the subsequent part of this paper.

Slightly different mechanism of crystallite growth at $700^{\circ} \mathrm{C}$ is found upon deposition of $2 \mathrm{ML}$ of Fe (Fig. 7). The shape of truncated pyramid is dominating for this coverage. The elongated forms, typical of $0.33 \mathrm{ML}$ coverage, are only sporadically met. Another striking feature is the strong influence of the vicinal structure on iron silicide crystallites nucleation. Crystallites grow mainly along the edges on the upper terraces. Practically none of them are found in the centre of terraces as it is observed for lower coverages. Such type of nucleation gives rise to the self-organization of iron silicide nanometer size crystallites, which may find practical implementation in nanoelectronics.

High-resolution image of a single crystallite grown upon deposition of $2 \mathrm{ML}$ $\mathrm{Fe}$ and subsequent annealing at $700^{\circ} \mathrm{C}$ is depicted in Fig. 8. The very regular shape of a truncated pyramid with six facets of type alternatively: (100) and (111) is clearly visible. The topmost layer again displays, like in the case of lower coverage, a $2 \times 2$ reconstruction with the distance between nearest atoms equal to $7.6 \AA$. The edges of the base are oriented along $[-110]$ directions of the very well 


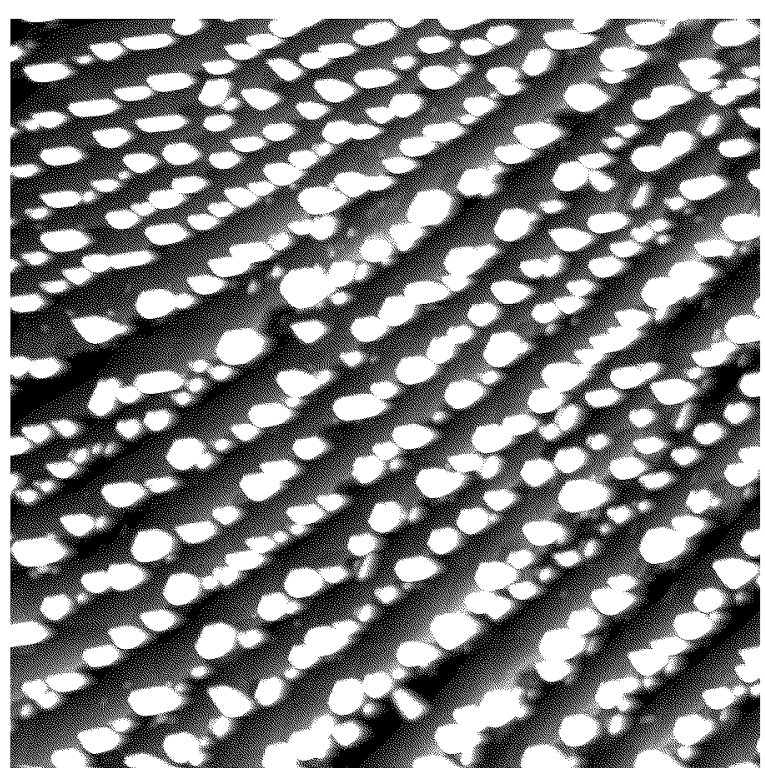

Fig. 7. Large scan $\left(1 \times 1 \mu \mathrm{m}^{2}\right)$ image of the self-organized iron silicide crystallites grown on the $\mathrm{Si}(111)$ vicinal surface upon deposition of $2 \mathrm{ML}$ Fe followed by annealing at $700^{\circ} \mathrm{C}$.

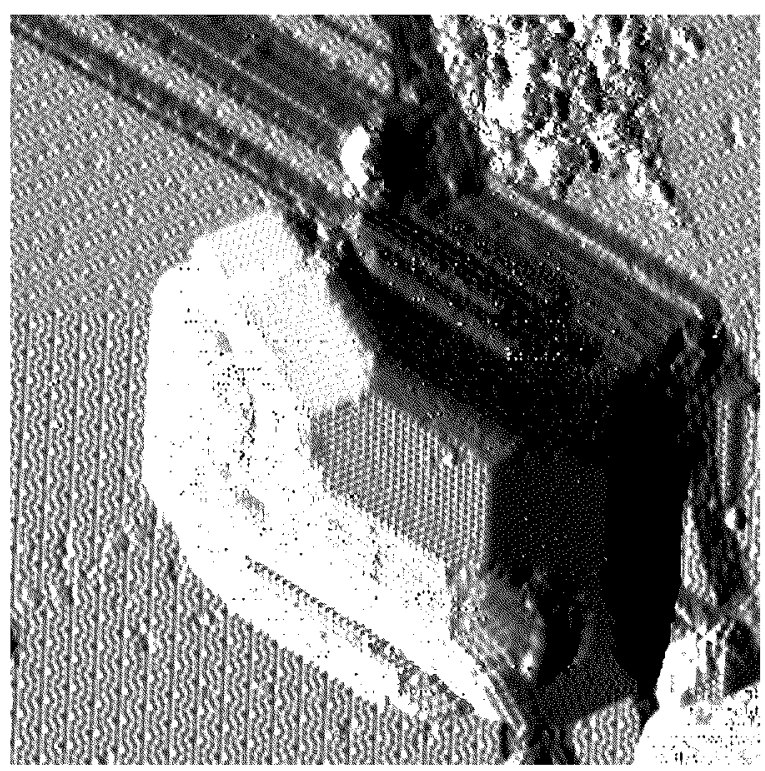

Fig. 8. STM derivative image of one of the elements of the self-organized array shown in Fig. 7. 
reconstructed $\operatorname{Si}(111) 7 \times 7$ substrate. Such correlation between the orientation of grown crystallites and the unit meshes of the substrate for both coverages is evidence that three-dimensional crystallites of iron silicide grow epitaxially on the Si substrate. Measured height of the crystallite $(68 \AA)$ in combination with the number of atomic planes, estimated on the basis of the topography of one of the facets (more than 30), favours the CsCl-type structure.

The diffusion processes proceeding on the surface at $700^{\circ} \mathrm{C}$ are responsible for agglomeration of Fe atoms (partially observed upon annealing at $400^{\circ} \mathrm{C}$ ), epitaxial growth of iron silicide crystallites and full reconstruction $7 \times 7$ of Si bare substrate, which is clearly visible in the background. It is reasonable to expect that all deposited Fe atoms are gathered in the volume of crystallized iron silicide. The temperature of $700^{\circ} \mathrm{C}$ is rather too low for re-evaporation of Fe atoms from the surface. The above assumption is taken into account in crystallite structure consideration in the subsequent part of the paper. The evidence of an intensive process of the Si surface etching, which supplies Si atoms for iron silicide formation, is very clear. The terraces of vicinal silicon surface have numerous, rounded edges corresponding to the height of double Si monolayer, which are not observed on the clean Si surface prior to the Fe deposition. In the vicinity of nucleated crystallites the substrate surface is flat without any dips around the crystallites. It means that the atoms necessary for iron silicide formation do not diffuse locally from sites in the vicinity of nucleating crystallites, but homogeneously from the entire surface. The localization of the big crystallites, grown upon deposition of 2 ML $\mathrm{Fe}$, along the edges of the upper terrace may result from the fact that this site is energetically favoured to supply high amount of substrate material, necessary for crystallite growth.

As mentioned earlier, different imaging of surface atoms is ascribed to spectroscopic effects. This is a characteristic feature of both: flat reconstructed surfaces formed upon deposition of $2 \mathrm{ML}$ Fe and subsequent annealing at $400^{\circ} \mathrm{C}$ as well as the surface and facets of iron silicide crystallites grown at $700^{\circ} \mathrm{C}$. In the constant current mode the tip of STM does not follow the geometric profile of the surface, but also probes an electronic local structure and these two contributions cannot be easily separated. However, on the basis of spectroscopic effects and results reported in other works, it seems that the differentiation between Fe and $\mathrm{Si}$ atoms is becoming possible.

A prevailing number of studies reveal that due to the decrease in surface energy the topmost layer of iron silicide is Si terminated [11, 14, 20, 21, 28]. The $\mathrm{Fe}-\mathrm{Si}$ bonding displays a homopolar nature, however in CsCl-type structure the transfer of approximately 0.5 electron charge per unit cell from Si atom to $d$ orbital of Fe atom is observed [2]. Thus, taking into account the non-uniform distribution of A-type and B-type atoms depending on the terminating layer (which can be treated as a preliminary stage formation of three-dimensional iron silicide crystallites), the following explanation seems to be credible. As mentioned earlier, Si 
atoms terminate the surface. They are very well distinguishable, because $s p$ orbitals are better imaged by STM than $d$ orbitals of Fe atoms. However these, which neighbour with $\mathrm{Fe}$ atoms in the layer underneath are slightly positively charged because of an electron transfer from Si towards Fe atom. When the occupied states are probed at negative bias of the tunneling junction, the intensity of the tunneling current decreases. In order to maintain a constant tunneling current, the STM tip approaches the surface and such type of surface atom is imaged as a "lower" one. Therefore the A-type atoms imaged as "lower" at negative bias are Si atoms adjacent to Fe atoms in subsurface layer whereas B-type ones are Si, which neighbour the same type of atoms underneath.

In order to study in detail the electronic structure of iron silicide crystallite surface, shown in Fig. 6, the measurements of the current imaging tunnelling spectroscopy (CITS) with atomic resolution have been performed. At positive bias the surface atoms are imaged in the same way. It is thus highly probable that the STM tip purely follows the topographic profile, i.e. the distance between the tip and the surface is kept constant over all atoms. In order to achieve the $I-V$ characteristics the bias of the tunneling junction has been swept from the positive to negative value. When the feedback loop is switched off to perform the spectroscopic measurements, the STM tip is positioned at the same height over the surface in every probed point. In such case the parameters of the junction barrier take the same values and the spatial variation of measured characteristics reflects merely electronic properties of the electrodes. Because the STM tip is cut from tungsten wire, which has a rather featureless electronic structure, the spatial variation, observed in current maps, should be associated only with the surface properties.

Constant current topography, simultaneously recorded current maps of the same area at various biases and $I-V$ as well as $\mathrm{d} I / \mathrm{d} V$ characteristics, measured at different sites of the surface are depicted in Fig. 9a-d. In topographic image (Fig. 9a) the bright vertical strip on the left side represents a part of the elongated iron silicide crystallite shown in Fig. 6 . Very well reconstructed $7 \times 7$ surface of the bare $\mathrm{Si}$ is clearly seen in the background. In the current image recorded at $0.39 \mathrm{~V}$ (Fig. 9b) the area of crystallite is imaged in black (in the current maps larger intensity of tunneling current is imaged brighter). It means that the intensity of the current flowing through iron silicide to the silicon substrate is lower in comparison with that directly tunnelling to the substrate. Substantially different map was recorded at the negative bias of $-1.1 \mathrm{~V}$ (Fig. 9c). The entire area of crystallite is imaged brighter than the uniform background of the bare silicon. Additionally the images of several surface atoms of crystallite are much brighter than the others. They correspond to the atoms, which are visible as "higher" ones in topography in Fig. 6. Approximately $25 \%$ of surface atoms of iron silicide crystallite display such a property. In accordance with the course of explanation given above, it is expected that the density of electronic states localized on such atoms takes a larger value. 
In order to find the differences in electronic structure, atomically resolved spectroscopic characteristics were measured. Figure $9 \mathrm{~d}$ depicts both $I-V$ and $\mathrm{d} I / \mathrm{d} V$ curves recorded at three various sites: bare silicon surface, bright and dark atoms of iron silicide crystallite. Each of them is averaged over at least ten runs obtained in various sites of the same type. As expected from the measurements of topography $I-V$ characteristics do not differ substantially in the range of positive biases. Merely for direct tunnelling to bare silicon the slope of the curve is higher
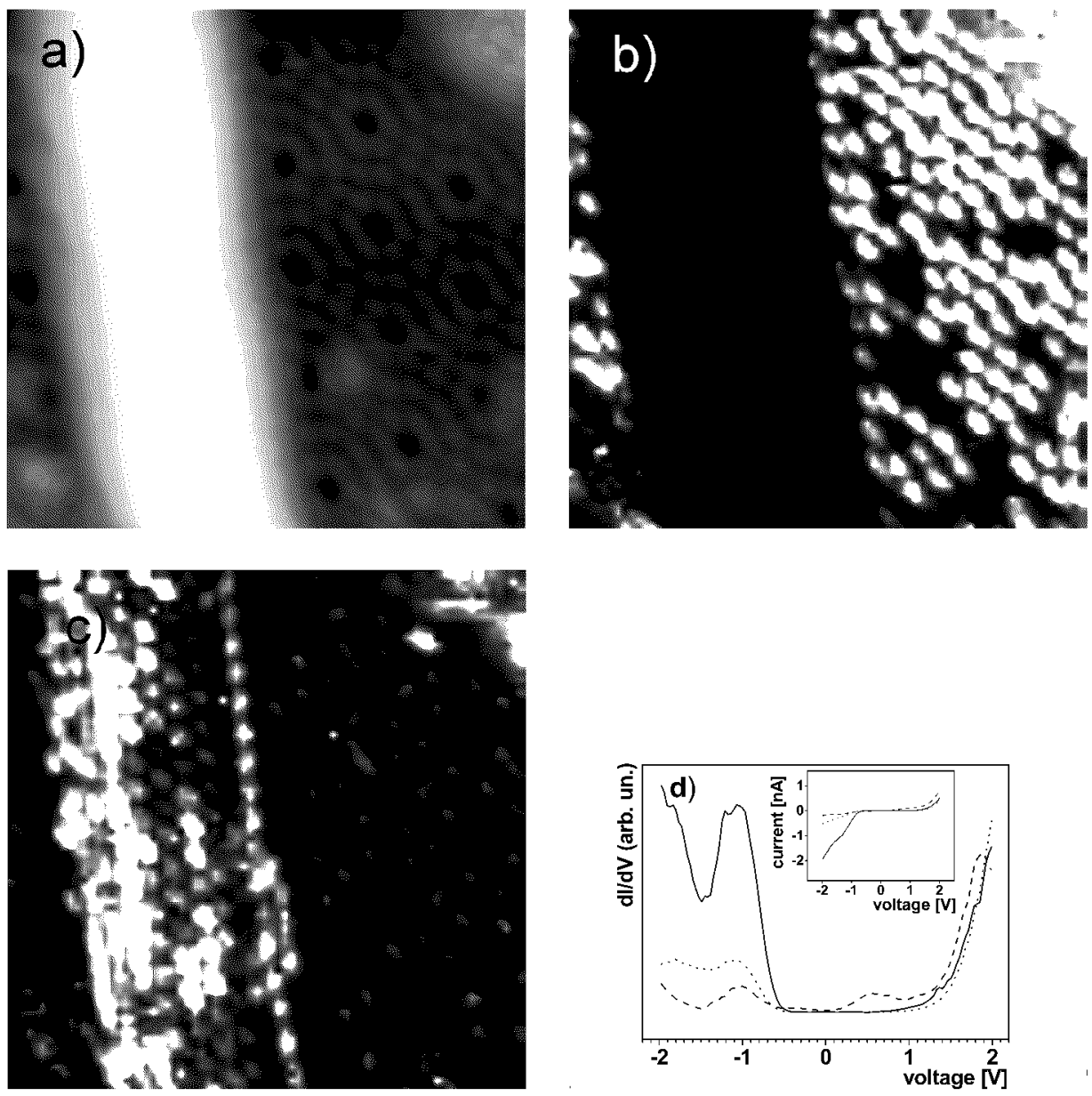

Fig. 9. (a) Recorded at +2.27 V STM topography image $\left(15 \times 15 \mathrm{~nm}^{2}\right)$ of the iron silicide nanocrystallite (bright vertical stripe on the left) grown on the bare Si(111) substrate after annealing of $0.33 \mathrm{ML} \mathrm{Fe}$ at $700^{\circ} \mathrm{C}$. Current map (CITS) of the same area obtained at: (b) $+0.39 \mathrm{~V}$ and (c) $-1.1 \mathrm{~V}$. (d) Averaged $\mathrm{d} I / \mathrm{d} V$ (in the insert $I-V$ ) characteristics recorded on: bare Si(111) surface (dashed line) and on iron silicide nanocrystal topmost layer: A-type atoms (dot line) and B-type atoms (solid line). 
between 0.4 and $1.2 \mathrm{~V}$. Much larger variations are observed below $-0.7 \mathrm{~V}$. Drastic increase in the tunnelling current is observed when the atoms of iron silicides, imaged in bright in the current map (Fig. 9c), are probed. The slope of $I-V$ characteristic for the remaining atoms of iron silicide crystallite is substantially lower, but still larger than that for pure silicon surface. Observed differences are emphasized in $\mathrm{d} I / \mathrm{d} V$ characteristics. Similarly to $I-V$ no differences are observed above $1 \mathrm{~V}$. Below this bias $\mathrm{d} I / \mathrm{d} V$ curves measured on iron silicide crystallite are flat whereas for Si substrate there is an apparent peak at $0.6 \mathrm{~V}$. The flat region, which may be associated with energy gap, extends down to $-0.5 \mathrm{~V}$ for all curves. Below this bias, where the occupied states are probed, the curve measured on B-type atoms drastically arises and two very distinct peaks appear at -1.1 and $-1.9 \mathrm{~V}$. Much weaker increase is observed for A-type atoms as well as for $\mathrm{Si}$ surface. The shape of $\mathrm{d} I / \mathrm{d} V$ characteristic reflects, at least qualitatively, the dependence of density of states on energy. A higher peak observed for B-type atoms in comparison with A-type is associated with the regular filling of electron orbitals in Si atom on the surface, when it is adjacent to the same type in the layer underneath. In the opposite case the transfer of electron charge towards Fe atom depletes the filling of orbitals in the topmost $\mathrm{Si}$ atom, which results in a weaker increase in the tunneling current with the bias and lower intensity of the peak in $\mathrm{d} I / \mathrm{d} V$ characteristic. The conducting and valence bands for positive and negative bias, respectively, are clearly highlighted. Much wider - in comparison to Si flat region around zero bias measured for iron silicide may suggest the presence of an additional tunneling barrier. Combination of the gathered data, i.e. metallic character of $(\mathrm{CsCl}) \mathrm{FeSi}$, diode-like drastic increase in the current intensity below $-0.7 \mathrm{~V}$ ( $I-V$ characteristics in Fig. 9d) and slightly larger apparent height of the same crystallite measured at negative bias in comparison with the height obtained at positive bias (e.g. from the profile comparison of the crystallite shown in Fig. 6), becomes consistent if the presence of the Schottky barrier at the interface between crystallites and $n$-type Si substrate is taken into account. Such an additional barrier reduces the tunnelling probability for the electrons flowing from the STM tip through the iron silicide crystallite to the substrate in comparison with the direct tunnelling to Si. The opposite bias decreases the efficiency of the barrier and tunnelling probability increases. In the constant current mode, in which topography is recorded, reduced tunnelling probability imposes the approach of the scanning tip over the surface, decreasing the apparent height of studied objects.

Detailed statistical analysis of the size of self-organized iron silicide crystallites grown on vicinal Si surface upon Fe deposition and subsequent annealing at $700^{\circ} \mathrm{C}$ enables an insight into their internal structure. The measured height, calibrated against the double Si monolayer step of $3.16 \AA$ in height, reveals the quantization close to $1.5 \AA$, typical of CsCl-type structure. This is clearly illustrated in Fig. 6, which shows the image of crystallite of $7.9 \AA$ in height consisting of five monoatomic layers. The height distribution in population of more than 80 


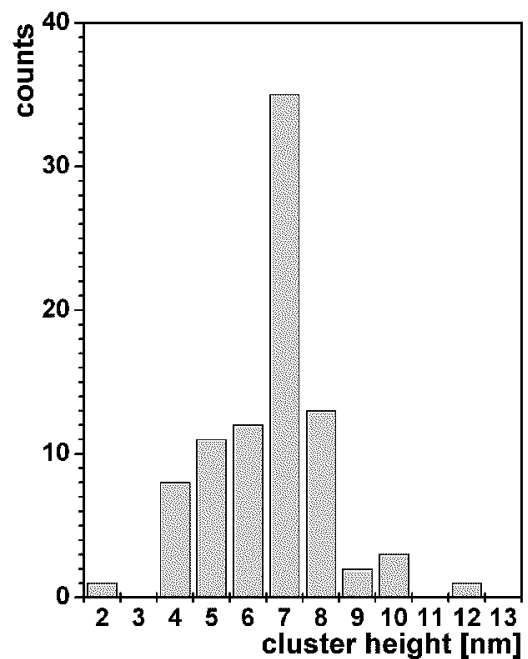

Fig. 10. Height histogram of iron silicide crystallites grown after annealing of $2 \mathrm{ML} \mathrm{Fe}$ at $700^{\circ} \mathrm{C}$.

randomly selected crystallites grown upon deposition of $2 \mathrm{ML}$ of Fe is shown in Fig. 10. Surprisingly, the obtained distribution has a non-Gaussian character. Instead, the crystallites of $7 \mathrm{~nm}$ in height dominate over the others, both larger and smaller in height. The above result may suggest that the investigated solid state reaction leads to the formation of a regular array of uniform in size iron silicide crystallites.

As mentioned earlier, the $2 \times 2$ reconstruction of iron silicide surface with the nearest neighbour distance between atoms equal to $7.6 \AA$ is characteristic of both $\mathrm{CsCl}(\mathrm{FeSi})$ and $\gamma-\mathrm{FeSi}_{2}$ phases. In order to identify the internal structure of grown crystallites, the ratio of their estimated volume to the volume of deposited iron is considered. The lattice parameter of CsCl-type phase is equal to $5.43 \AA$. The elementary cell of this phase contains eight atoms of $\mathrm{Fe}$ and Si. The lattice parameter of $\gamma-\mathrm{FeSi}_{2}$ compound is comparable and equal to $5.39 \AA$, however the elementary cell consists of eight $\mathrm{Si}$ atoms and four $\mathrm{Fe}$ atoms. The $\mathrm{CsCl}$ vacancy type structure, in which the iron atoms are substituted by vacancies, is the intermediate one between the two phases described above. Under reasonable assumption that the temperature of $700^{\circ} \mathrm{C}$ is too low for re-evaporation of $\mathrm{Fe}$ atoms from the surface it is expected that all deposited Fe atoms are built in the iron silicide crystallites. Thus the ratio of compound volume to the bulk-like volume of deposited iron is equal to 1.7 and 3.3 for $\mathrm{CsCl}$ - and $\gamma$-type structures, respectively. The volume of grown crystallites may be approximately estimated on the basis of their height distribution and the fraction of the sample's area, which is covered with iron silicide. The statistics for both coverages are shown in Table. Obtained results 
TABLE

Parameters applied for estimation of iron silicide crystallite structure.

\begin{tabular}{c|c|c|c|c}
\hline \hline $\begin{array}{c}\text { Coverage } \\
{[\mathrm{ML}]}\end{array}$ & $\begin{array}{c}\text { Fe layer thickness } \\
{[\mathrm{nm}]}\end{array}$ & $\begin{array}{c}\text { Occupied surface } \\
{[\%]}\end{array}$ & $\begin{array}{c}\text { Crystallite mean height } \\
{[\mathrm{nm}]}\end{array}$ & \begin{tabular}{c}
$V_{\mathrm{FeSi}} / V_{\mathrm{Fe}}$ \\
\hline 0.33
\end{tabular} \\
0.048 & 11 & 0.8 & 1.83 \\
2 & 0.29 & 13 & 6.6 & 2.9
\end{tabular}

clearly confirm that the grown iron silicide crystallites for both coverages have CsCl-type structure, however the smaller ones are more compact with composition close to $1: 1$, whereas in bigger ones a certain amount of vacancies is present. Inferred internal structure of crystallites coincides well with the STM images of their surface. In the case of smaller crystallites the different STM imaging of atoms is frequent, which is in contrast to bigger ones, where the surface atoms are imaged in the same way. It suggests that in smaller crystallites, where the contribution of atoms located on the surface and in subsurface layers is much higher and the fraction of $\mathrm{Fe}$ atoms approaches $50 \%$, the presence of $\mathrm{Fe}$ atoms in the vicinity crystal surface is highly probable. The surface of big crystallites, in which the relative number of surface atoms is much lower, is probably terminated with a double Si layer, as predicted by theoretical models [14, 20], and in consequence imaged homogeneously.

\section{Conclusions}

The solid state reaction proceeding on Si vicinal surface upon deposition of Fe and subsequent annealing results in the growth of iron silicide crystallites. The initial stage of nucleation is found already upon annealing at $400^{\circ} \mathrm{C}$. At $700^{\circ} \mathrm{C}$ metallic, three-dimensional iron silicide crystallites of CsCl-type structure grow in the form of truncated pyramids on bare $7 \times 7$ reconstructed Si surface. Depending of Fe coverage they take different shape, size, and location. The smaller are elongated and nucleate homogeneously on the surface. The bigger ones decorate the edges of the upper terraces giving rise to formation of a regular array of nanometer in size metallic dots on the semiconducting substrate. They show a tendency to grow in uniform size of $7 \mathrm{~nm}$ in height. Basing on spectroscopic effects, the composition and structure of the crystallites' surface is proposed. Statistical analysis reveals that in bigger crystallites the contribution of vacancies in CsCl-type structure is higher in comparison with smaller ones. The properties of the interface between metallic crystallite and semiconducting substrate resemble Schottky barrier behaviour.

\section{Acknowledgments}

This work was partially supported by the State Committee for Scientific Research under the project R-34. 


\section{References}

[1] D. Leong, M. Harry, K.J. Reeson, K.P. Homewood, Nature 387, 686 (1997).

[2] H. von Känel, K.A. Mäder, E. Müller, N. Onda, H. Sirringhaus, Phys. Rev. B 45, 13807 (1992).

[3] H. Sirringhaus, N. Onda, E. Müller-Gubler, P. Müller, R. Stalder, H. von Känel, Phys, Rev. B 47, 10567 (1993).

[4] C. Pirri, M.H. Tuilier, P. Wetzel, S. Hong, B. Bolmont, G. Gewinner, R. Cortès, O. Heckmann, H. von Känel, Phys. Rev. B 51, 2302 (1995).

[5] K.L. Whiteaker, I.K. Robinson, C. Benson, D.M. Smilgies, N. Onda, H. von Känel, Phys. Rev. B 51, 9715 (1995).

[6] M. Fanciulli, C. Rosenblad, G. Weyer, A. Svane, N.E. Christensen, H. von Känel, Phys. Rev. Lett. 75, 1642 (1995).

[7] M. Fanciulli, G. Weyer, A. Svane, N.E. Christensen, H. von Känel, E. Müller, N. Onda, L. Miglio, F. Tavazza, M. Celino, Phys. Rev. B 59, 3675 (1999).

[8] J.M. Gallego, J. Alvarez, J.J. Hinarejos, E.G. Michel, R. Miranda, Surf. Sci. 251-252, 59 (1991).

[9] N. Motta, A. Sgarlata, G. Gaggiotti, F. Patella, A. Balzarotti, M. de Crescenzi, Surf. Sci. 284, 257 (1993).

[10] J.H. Oh, S.K. Lee, K.P. Han, K.S. An, C.Y. Park, Thin Solid Films 341, 160 (1999).

[11] A.L. Vazquez de Parga, J. de la Figuera, C. Ocal, R. Miranda, Europhys. Lett. 18, 595 (1992).

[12] U. Kafader, M.H. Tuilier, C. Pirri, P. Wetzel, G. Gewinner, D. Blomont, O. Heckmann, D. Chandesris, H. Magnan, Europhys. Lett. 22, 529 (1993).

[13] X. Wallart, J.P. Nys, C. Tételin, Phys. Rev. B 49, 5714 (1994).

[14] A. Mascaraque, J. Avila, C. Teodorescu, M.C. Asensio, E.G. Michel, Phys. Rev. $B$ 55, R7315 (1997).

[15] N. Jedrecy, A. Waldhauer, M. Sauvage-Simkin, R. Pinchaux, Y. Zheng, Phys. Rev. $B$ 49, 4725 (1994).

[16] J.J. Hinarejos, G.R. Castro, P. Segovia, J. Alvarez, E.G. Michel, R. Miranda, A. Rodriguez-Marco, D. Sánchez-Portal, E. Artacho, F. Ynduráin, S.H. Yang, P. Ordejón, J.B. Adams, Phys. Rev. B 55, R16065 (1997).

[17] Le Thanh Vinh, J. Chevrier, J. Derrien, Phys. Rev. B 46, 15946 (1993).

[18] K.A. Mäder, H. von Känel, A. Baldereschi, Phys. Rev. B 48, 4364 (1993).

[19] U. Starke, J. Schardt, W. Weiss, W. Meier, C. Polop, P.L. de Andres, K. Heinz, Europhys. Lett. 56, 822 (2001).

[20] M. Sauvage-Simkin, N. Jedrecy, A. Waldhauer, R. Pinchaux, Physica B 198, 48 (1994).

[21] U.K. Köhler, J.E. Demuth, R.J. Hamers, Phys. Rev. Lett. 60, 2499 (1988).

[22] W. Raunau, H. Niehus, G. Comsa, Surf. Sci. Lett. 284, L375 (1993).

[23] J. Junquera, R. Weht, P. Ordejón, Surf. Sci. 482-485, 625 (2001).

[24] A. Wawro, unpublished. 
[25] U. Starke, W. Weiss, M. Kutschera, R. Bandorf, K. Heinz, J. Appl. Phys. 91, 6154 (2002).

[26] W. Raunau, H. Niehus, T. Schilling, G. Comsa, Surf. Sci. 286, 203 (1993).

[27] J. Chevrier, V. Le Thanh, S. Nitsche, J. Derrien, Appl. Surf. Sci. 56-58, 438 (1992).

[28] S.A. Chambers, S.B. Anderson, H.W. Chen, J.H. Weaver, Phys. Rev. B 34, 913 (1986). 